\title{
Research of the Snatch and Holding up Safety Automatic Control System for the Farm Grasping Grass Machine
}

\author{
Zhen-hang Cao* ${ }^{1}$, Guan-feng $\mathrm{Li}^{2}$ and Zhong-Xu Dou ${ }^{3}$ \\ ${ }^{1,2,3}$ College of Mechanical and Electrical Engineering, Henan Agricultural University, Zhengzhou, \\ Jinshui district, China \\ ${ }^{*}$ Corresponding author
}

Keywords: The Grasping Grass Machine, Snatch and Holding up Safety Security, Fall Forward, Automatic Control System

\begin{abstract}
This paper introduced tilting accident phenomenon of the farm grasping grass machine in the practical application. Through to analyze overturn accident of instantaneous critical stress state at work, this paper put forward a basic idea that can control and prevent the overturn accident, designed the snatch and holding up safety automatic control system. It can detect the pressure changes of lifting hydraulic by installed pressure sensor in the lifting oil cylinder, and with the aid of weight-pressure mathematical model, cooperate with dynamic testing technology measures and corresponding measures of hardware, to realize the dynamic real-time monitoring of snatch and holding up weight. That the function of accumulated and recorded load, the early warning of overweight and accident prevention about falling down are realized by the system overall structure and software design.
\end{abstract}

\section{Introduction}

As a key equipment of crop straw resource utilization, the farm grasping grass machine has been the focus of attention and developed rapidly in recent years in our country, and its development and popularization application have provided strong support for effectively solving collection and utilization problem of the crop straw after the crop had been harvested in China ${ }^{[1]}$. With the continuous expansion of the market using farm grasping grass machines, the basic performance of the product is gradually improved and improved. However, there are still some problems in use, and one of the main problems is the initiative to be further improved to prevent rollover accidents remains. Therefore, how to improve the safety of its own structural design to meet the stringent national policies and regulations on the safety requirements of the equipment operation has become the focus of research. In this connection, this paper puts forward a snatch and holding up safety automatic control system of the grasping grass machine, in order to solve the problems that the machine down accidents caused by snatch overweight in working .

\section{Analysis of Snatch and Holding up Stability for the Grasping Grass Machine}

The most common accidents of the grasping grass machine is fall down to the direction of the goods, and caused economic losses and casualties, when the machine working the rear wheel is cocked by the goods due to snatch the object more than the rated quality.

Figure 1 is the schematic diagram of the grasping grass machine in working. In the figure, the $\mathrm{M}$ is the total mass of the machine, the $m$ is the overall quality of the goods and gripper head. As the figure shows, when the grasping grass machine grabbed the overloaded goods to fall, the ground pressure of the rear wheel diminishes gradually in this process. And when the ground pressure of the rear wheels reduces to zero, the rear wheels will leave the ground. Before at this time, if the grasping grass machine does not stop lifting, the rear wheels will be prized up by the goods, and the grasping grass machine will be rotated and fell forward around the center of front wheel. At this point if the grasping grass machine remind the driver to stop the snatch by alarm, or by automatic control device locks the lifting hydraulic cylinder, cannot make it continue lifting, the accident will be effectively avoided. 


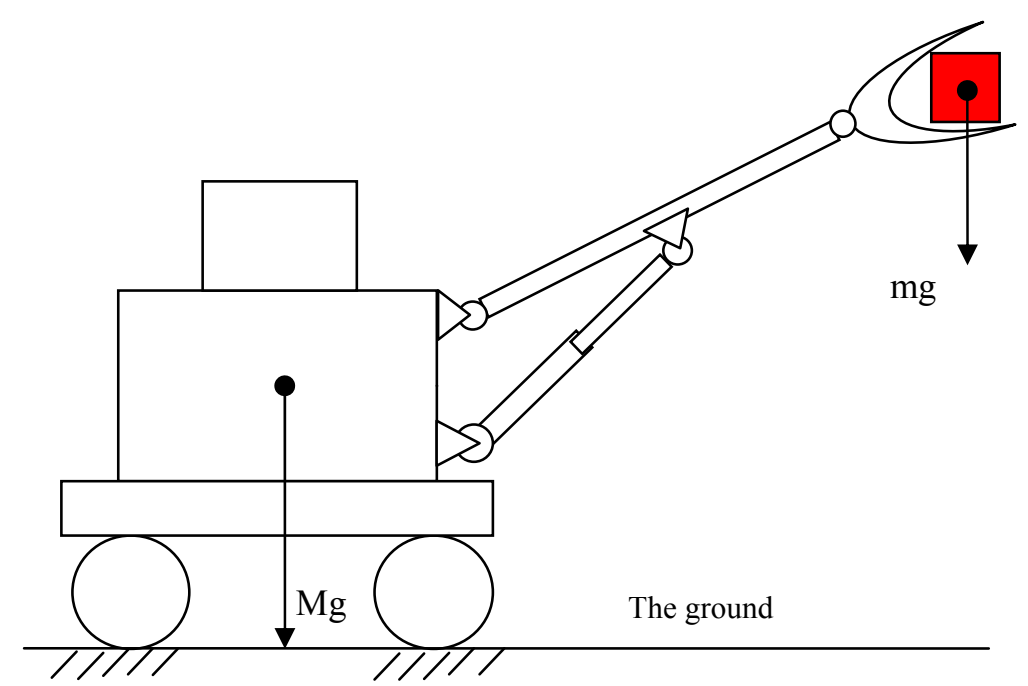

Figure 1. Schematic diagram of the grasping grass machine in working

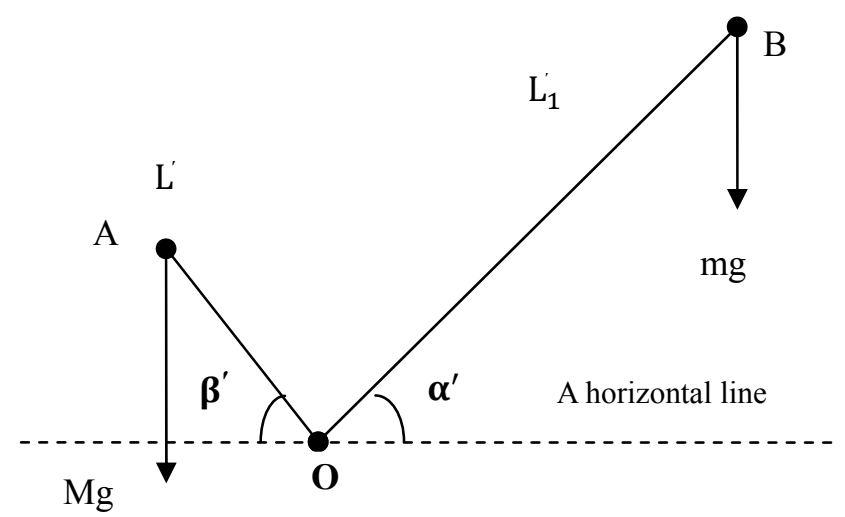

Figure 2. Critical state mathematical model diagram of the grasping grass machine

Figure 2 is the critical state force balance diagram that the grasping grass machine lifting goods. The $\mathrm{A}$ is the center of gravity of the grasping grass machine, the B is the center of gravity of the goods, the $\mathrm{O}$ is the front wheel center, the $\mathrm{M}$ is the quality of the grasping grass machine works, the $\mathrm{m}$ is the quality of the goods, sets the distance of AO to L', the distance of BO is L1', the Angle of $\mathrm{AO}$ and horizontal line is $\beta$ ', the Angle of "BO" and horizontal line is $\alpha$ ', the $\mathrm{g}$ is the gravitational acceleration.

When the grasping grass machine dumped, it will be influenced by its own gravity and gravity of goods. They make up the lever that is to the grasping grass machine's front wheel center " $\mathrm{O}$ " as the fulcrum. Thus, we can get the formula from the lever balance principle:

$$
M g L^{\prime} \cos \beta^{\prime}=\operatorname{mgL}_{1}^{\prime} \cos \alpha^{\prime}
$$

When $m=\frac{L^{\prime} \cos \beta^{\prime}}{L_{1}^{\prime} \cos \alpha^{\prime}} M$, the grasping grass machine reached a tipping point.

If the force arm of goods gravity to point $\mathrm{O} \mathrm{mgL}_{1}^{\prime} \cos \alpha$ 'less than the force arm of the grasping grass machine gravity to point $\mathrm{O} M g L^{\prime} \cos \beta$, the grasping grass machine will not fall forward.

If the force arm of goods gravity to point $\mathrm{O} \mathrm{mgL}_{1}^{\prime} \cos \alpha^{\prime}$ more than the force arm of the grasping grass machine gravity to point $\mathrm{O} M g L^{\prime} \cos \beta^{\prime}$, the grasping grass machine will fall forward.

\section{The Function of Snatch and Holding up Safety Automatic Control System}

Grapple machine snatch and hoiling up safety automatic control system is used to monitor the 
snatch weight in the work process of grass cutting machine, and to prevent the tilting lifting turn accident caused by overweight and forcibly lift. It has two main functions, one is to achieve the work volume statistics .More concretely, the system weighted the good quality of each grab in the work process, and then cumulative calculation quality of the goods before grabbed, through the accumulation of statistical records obtain the work volume of the grasping grass machine all day. The other is to achieve security early warning and automatic control. When the grab goods exceed the rated snatch amount, the alarm device will cut off the hydraulic lifting cylinder temporarily, stopping the lifting action, and reminding the operator choose the snatch object again, to avoid leaning over accidents.

\section{The Realization of the Function for Weighing and Recording}

(1)Quality acquisition scheme. Weighing is the foundation of snatch and holding up safety automatic control system. It has many methods to get the weight information. Using the method that installed the pressure sensor on oil cylinder of grasping grass machine's mobile arm, by monitoring the pressure changes of the lifting oil cylinder in the work, establish a mathematical model of the relation between oil cylinder pressure and snatch the goods' weight. Then, we can get the quality of goods, and this method is relatively simple and feasible [2]. Principle of this method is through establishing the mathematical model of weight and pressure, determine the relationship between snatch object quality and oil cylinder pressure. It is using the sensor measured cylinder pressure in lifting oil cylinder, thus indirectly get the quality of goods. But it still needs to solve three problems in the actual process of weighing: (1) claw is moving, has the certain impulse, can cause certain error to measurement results (need to complement in the program settings); (2) the oil pressure is analog signal and it is not stable, need fast modulus conversion; (3) in the dynamic case, the correct solution still needs the support of hardware and software.

A



Figure 3. Weight-pressure mathematical model diagram of the grasping grass machine

(2) The mathematical model of weight and pressure. As shown in figure 3 is the weight-pressure mathematical model of the grasping grass machine operations: Lifting arm is a four connecting rods and a mechanics geometric structure, many factors which influence the cylinder pressure. Such as lifting weight, the angle of the lifting arm, the running speed of claw, etc. When the grass machine arm claw snatching and holding up the same goods in different acceleration, oil pressure will present different values of number. Therefore, the corresponding snatch quality $\mathrm{m}$ is calculated by measuring the pressure change in the oil cylinder.

In the figure: $A B$ is the movable arm length of $\mathrm{L} . \mathrm{A}$ is the hinge point of the movable arm. $\mathrm{B}$ is the center of goods' gravity. $\mathrm{CD}$ is the lifting oil cylinder of the movable arm. $\mathrm{C}$ is the joint of lifting oil cylinder and the body. $\mathrm{D}$ is the join of movable arm and oil cylinder. Assume $\mathrm{A}$ and $\mathrm{C}$ are in the same vertical surface, $h$ is the distance between $A$ and C.ais the angle of the movable arm and the horizontal clip at some point. $\beta$ is the angle of oil cylinder and vertical clip. $\mathrm{F}$ is the oil pressure forced movable arm. Because each component deformation of the grasping grass machine is small 
in working process, Therefore, assume that all the components are the rigid body [3].

Static situations:

When the lifting arm stationary, analysis of $\mathrm{AB}$, torque balance equation as follows:

$$
\operatorname{mgL} \cos \alpha=\text { Fh } \sin \beta
$$

So $\mathrm{m}=\frac{\mathrm{h} \sin \beta}{\mathrm{gL} \cos \alpha} \mathrm{F}$. The $\frac{\mathrm{h} \sin \beta}{\mathrm{gL} \cos \alpha}$ is the fixed value, so the oil pressure $\mathrm{F}$ is a linear relationship with lifting weight $\mathrm{m}$ at this time.

Dynamic circumstances:

(1)When the lifting arm movement at a constant speed, the instantaneous oil pressure (the sensor instantaneous sampling values) is a linear relationship with the quality of lifting goods. And the relationship equation is the same as the stationary: $\mathrm{m}=\frac{\mathrm{h} \sin \beta}{\mathrm{gL} \cos \alpha} \mathrm{F}$.

(2)When the lifting arm variable speed rise, the oil pressure $F$ is a nonlinear relationship with lifting weight $\mathrm{m}$ at this time. By the rotation theorem concluded that:

$$
\text { Fh } \sin \beta-\text { GL } \cos \alpha=\mathrm{Ja}
$$

In the equation, $\mathrm{J}$ is the moment of inertia of the goods for A point $\left(\mathrm{J}=\mathrm{mL}^{2}\right)$, and the a is the angular acceleration of the goods[4], so

$$
\mathrm{m}=\frac{\mathrm{h} \sin \beta}{\mathrm{gL} \cos \alpha} \mathrm{F}-\frac{\mathrm{Ja}}{\mathrm{gL} \cos \alpha} .
$$

From this consequence, we know that the " $\mathrm{a}$ " is changing, so the relationship between $\mathrm{F}$ and $\mathrm{m}$ is nonlinear. But we can eliminate the influence of the angular acceleration by sensor bridge circuit, and this time the $\mathrm{F}$ is the approximate linear relationship with the $\mathrm{m}$.



Figure 4. Detection technology structure

(3) The dynamic detection technology measures. In order to eliminate the influence of the acceleration and obtain the stability of the output signal, sensor's output signal needs filter in circuit design. On the data processing of the single chip microcomputer, the data sequence is searched by stationary signal further processing and filtering technology.

Dynamic testing technology structure is as shown in figure 4. Working principle is: when the power $-5 \mathrm{~V} \sim 5 \mathrm{~V}$ adds to the sensor under detection, the bridge circuit of the sensor output the electrical signals about $-75 \mathrm{mV} \sim 75 \mathrm{mV}$. The signal passes the differential amplifier low-pass filter, get $-1.5 \mathrm{~V} \sim 1.5 \mathrm{~V}$ analog electrical signals. To pass $\mathrm{A} / \mathrm{D}$ converter that under control in the single-chip microcomputer, get the corresponding digital quantity with the detection quality, through single-chip microcomputer to calculate, transform BCD code, and then send it to the computer for computing.

(4) The selection of the sensor. Types of sensors are so many, different types of sensors have different application areas, using range and features. Resistance strain sensor has many advantages compared with other types of sensor. These benefits include: High precision, wide measurement range, long service life, stable and reliable performance, good frequency response characteristics, simple structure, small size, light weight, low cost and it can work under the bad environment, etc. 
In view of the stability, accuracy, sensitivity, life, etc, system selects the resistance strain sensor (ZQ - PM flat diaphragm type pressure transmitter). Consider to the hydraulic oil pressure and temperature range of the grasping grass machine, sensor pressure is $20 \mathrm{Mpa}$ on the system, temperature range is $0-100 \square$.

\section{The Realization of the Function for Security Early Warning}

The safety warning of the grasping grass machine is actualized by that the sensors installing on the lifting oil cylinder detects the pressure change of the lifting oil cylinder and output the corresponding electrical signal, this signal will be send to the SCM operation analysis after amplification, filtering and A/D conversion, and then sent to the PC shows that the quality of the goods (it bases on snatch weighing), and compared with rated value to judge whether the quality of the snatch exceed the limits.

The rated snatch quality of the grasping grass machine is " $\mathrm{m}_{\mathrm{r}}=\mathrm{Km}_{\mathrm{s}}$ ". In the formula: $\mathrm{m}_{\mathrm{r}}$ is the rated snatch quality; $\mathrm{K}$ is the weight utilization coefficient, the range of the $\mathrm{K}$ of the wheeled grasping grass machine is $0.25 \sim 0.30 ; \mathrm{m}_{\mathrm{s}}$ is the whole machine quality of the grasping grass machine[7].In order to ensure the work safety of the grasping grass machine, We choose $\mathrm{m}_{\mathrm{r}}=$ $0.25 \mathrm{~m}_{\mathrm{s}}$ as the rated snatch quality. If the grasping grass machine grabs the quality of the goods' $\mathrm{m} \leq 0.25 \mathrm{~m}_{\mathrm{s}}$ ', the grasping grass machine will work normally. When the grasping grass machine grabbed the quality of the goods ' $\mathrm{m}>0.25 \mathrm{~m}_{\mathrm{s}}$ ', the computer instructions cut off the hydraulic circuit through electromagnetic valve, stop lifting, and trigger the alarm device, remind the driver to select snatch, implement security early warning and prevent accidents.

\section{The Overall Structure and Software Design}

\section{The General Structure of Snatch and Holding up Safety Automatic Control System}

The grasping grass machine's snatch and holding up safety automatic control system was composed of the signal acquisition processors, the middle processor (PC) and the control actuator. In addition, the system also includes power module (it provides a stable voltage for the system, and it makes the whole system normal and stable), set display, control and alarm functions in one. The figure 5 is the structure of snatch and holding up safety automatic control system.

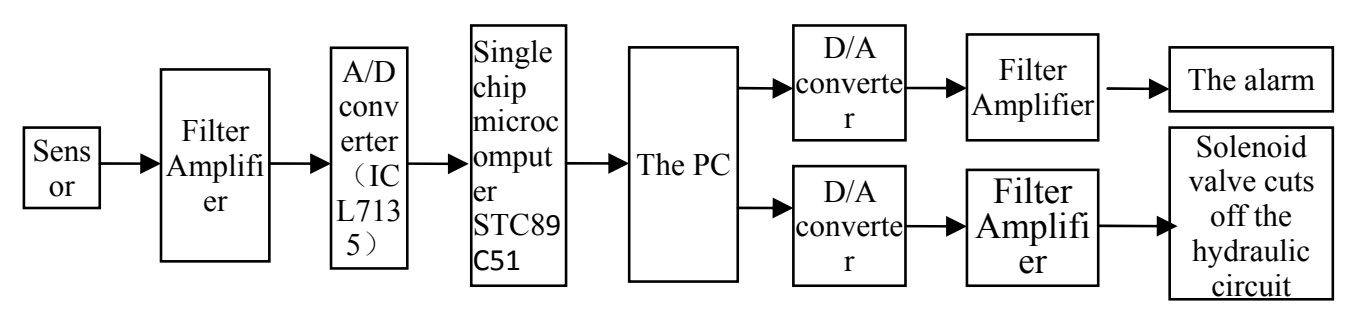

Figure 5. Snatch safety automatic control system structure

\section{The Design of the Software}

The core of the snatch and holding up safety automatic control system of grasping grass machine adopts single-chip microcomputer STC89C51, all programs are solidified in FLASH, and a computer is used to store the testing data. The main function of the main program are: system initialization and initial condition set, distinguish work function, sampling digital filtering, sensor zero point automatic tracking, etc. The process is shown in figure 6 . 


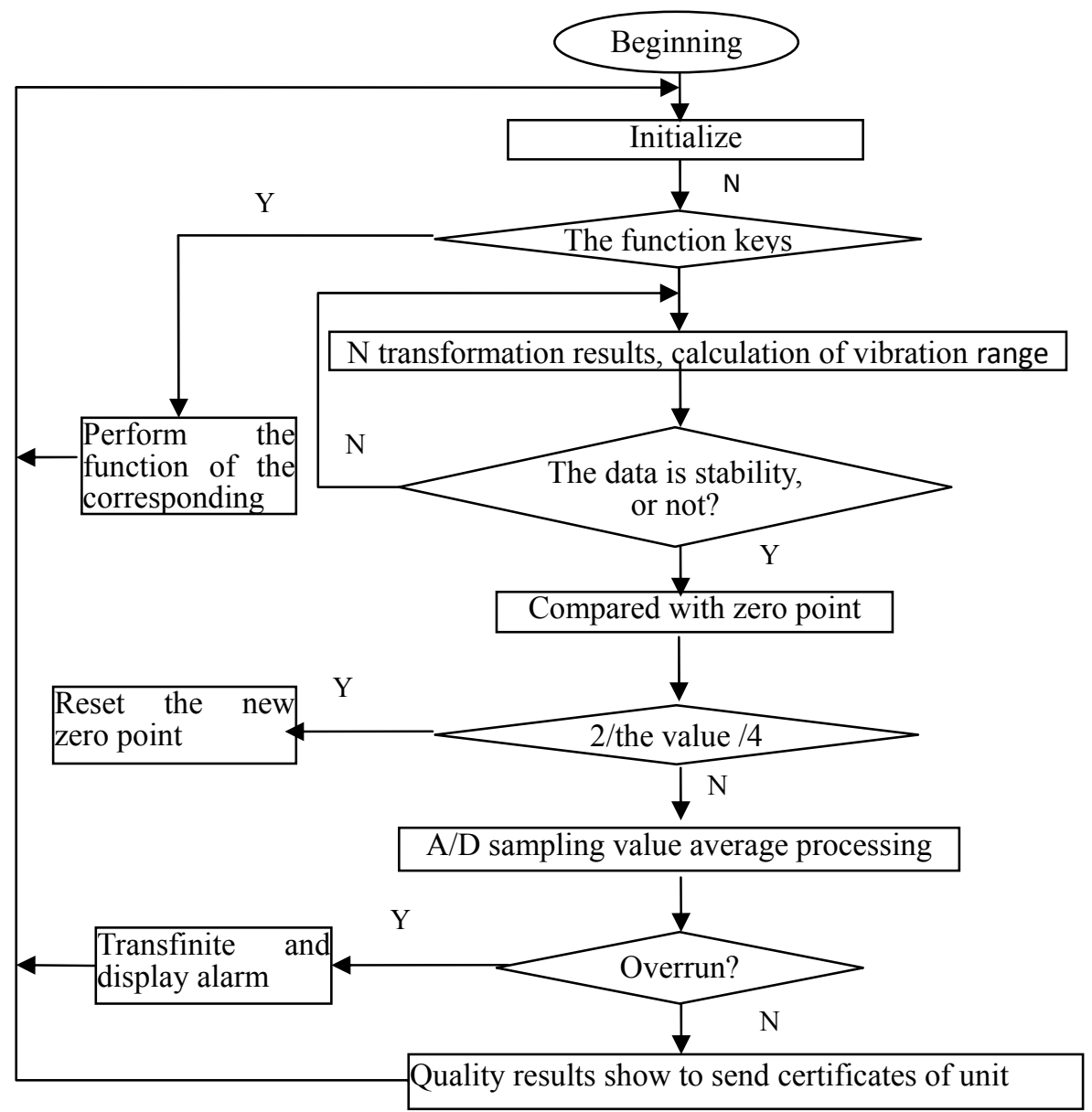

Figure 6. Main program flow of snatch safety automatic control system

(1) On data acquisition and data processing, digital filtering technology and spike pulse rejecting technology is used to eliminate the impact acceleration in the aspect of vibration for the detection sensor. The basic idea of the design is the dynamic stability. Specific is to electrical signal of the sensor output sampling continuously in a period, calculate the vibration amplitude. If the vibration amplitude is meet the requirements of the vibration tolerance, the average processing, otherwise, must carry on the new sample, until it meets the requirements within the number of the limit.

(2) The show result lock, quality test results into the computer display by single-chip microcomputer, the display results in a constant state.

(3) Use the software program to automatically tracking zero drift of the sensor. Due to the influence of temperature drift and time drift, the sensor's zero often drift phenomenon, the measures are not taken, will affect the accuracy of measurement. The quality sampling adopted the software automatic tracking measures. The basic idea of the program design is to zero quality value sampling, tracking the change, changes to reset the zero value.

\section{Conclusions}

With the increase rate of new rural construction and national attention to the environmental protection, the agricultural renewable resources utilization will further enhance. As the key equipment of comprehensive utilization for crop straw, the grasping grass machine will to a higher degree developing. Given that there are many accidents in the process of production, its operation safety becomes the major factor that impedes the spread. The snatch and holding up safety automatic control system of the grasping grass machine is proposed in this paper has the function of work records and security early warning, and it provides the reference to solve the accident problem that due to snatch the overweight fell in the actual production. I hope it would promote the farm 
grasping grass machine's technology improvement and development.

\section{Reference}

[1] Cao Zhenhang, Li guanfeng, Dou zhongxu, Zhang Yi, Zhang zhigang. Research of the Intelligent Control Development Trends of Farm Grasping Grass Machine [J]. Agricultural Equipment and Vehicle Engineering, 2016 (10).

[2] Wang Kai, loader intelligent dynamic weighing system design [D]. Zhongyuan Engineering College, 20110112.

[3] R.C.Hibbeler. Engineering mechanics----dynamics [M]. Beijing: Electronic Industry Press, 2006:473-506.

[4] Liu Kezhe. Physics (upper part) [M]. Beijing: Higher Education Press, 1999:101-114.

[5] Hu xiangdong, Liu Jingcheng. Sensing technology [M]. Chongqing: Chongqing University Press, 2006:48-63.

[6] Zhu Ninghe. Loader Intelligent weighing instrument design [D]. Southwest Jiaotong University, 20080301.

[7] Yang Zhanmin, Wang Zhiming, Zhang Chunqiu, etc. Wheel loader [M]. Beijing: Chemical Industry Press, 2006:16-17 